\title{
Blood Concentrations of Homocysteine and Methylmalonic Acid among Demented and Non-Demented Swedish Elderly with and without Home Care Services and Vitamin $B_{12}$ Prescriptions
}

\author{
Nils-Olof Hagnelius $^{\text {a }} \quad$ Lars-Olof Wahlund $^{c}$ Jörn Schneede ${ }^{d}$ \\ Torbjörn K. Nilsson ${ }^{b}$

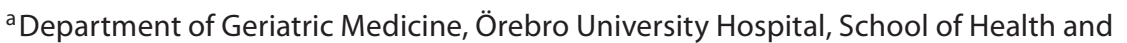 \\ Medical Sciences, Örebro University, and ${ }^{b}$ Department of Laboratory Medicine, Division \\ of Clinical Chemistry, Örebro University Hospital, Örebro, ' Department of Neurobiology, \\ Care Sciences and Society, Section of Clinical Geriatrics, Karolinska Institutet, Stockholm, \\ and ${ }^{d}$ Department of Pharmacology and Clinical Neuroscience, Division of Clinical \\ Pharmacology, University Hospital of Northern Sweden, Umeå, Sweden
}

\section{Key Words}

Homocysteine $\cdot$ Dementia $\cdot$ Vitamin $\mathrm{B}_{12} \cdot$ Folate $\cdot$ Home care service

\begin{abstract}
Background and Aims: Total plasma homocysteine (tHcy) has been suggested as a risk factor of dementia. Our aim was to investigate potential differences in thcy status in relation to the prescription of vitamin $B_{12}$ and dementia diagnosis. We examined whether vitamin $B_{12}$ prescriptions, a family history of dementia, or the need for home care service might be associated with thcy values. Methods: A cross-sectional monocenter study comprising 926 consecutive subjects attending our Memory Care Unit was conducted. Results: Demented subjects being prescribed vitamin $B_{12}$ had higher serum vitamin $B_{12}(p=0.025)$ but also higher tHcy $(p<0.001)$ and serum methylmalonate $(p=0.032)$, and lower serum folate $(p<0.001)$ than those who did not receive vitamin $B_{12}$ prescriptions. tHcy levels were significantly higher in non-demented subjects receiving home care service $(p=0.007)$. This group also had lower serum albumin (dementia: $p<0.001$; non-dementia: $p=0.004$ ). There was no difference in renal function (estimated glomerular filtration rate) in demented or non-demented subjects with or without vitamin $B_{12}$ prescriptions (dementia with/without vitamin $B_{12}$ prescription: $p=0.561$; non-dementia with/
\end{abstract}


without vitamin $B_{12}$ prescription: $p=0.710$ ). Conclusion: Despite vitamin $B_{12}$ prescriptions, demented subjects had higher tHcy and methylmalonate values. The elevated metabolite values could not be explained by differences in renal function. Thus, elderly subjects on vitamin $B_{12}$ prescription appear to have unmet nutritional needs.

Copyright $\odot 2012$ S. Karger AG, Base

\section{Introduction}

There has been a growing interest in the role of vitamin deficiencies in relation to dementia. Vitamin $B_{12}$ was among the first vitamins being investigated in relation to neuropsychiatric disease, and already in the mid-19th century, Addison [1] described neuropsychiatric symptoms in patients with pernicious anemia ('the mind occasionally wanders'). There are reports on improvement of mental symptoms upon vitamin $B_{12}$ supplementation $[2,3]$. In addition to insufficient vitamin $B_{12}$ status, folate deficiency has also been discussed in the pathogenesis of dementia [4-8]. A prospective Canadian study among older persons found that low serum folate was associated with a higher risk of cerebrovascular events including vascular dementia and death because of stroke [9].

The essential amino acid methionine, together with vitamin $B_{12}$ and folate, is centrally involved in the one-carbon metabolism. Methionine serves in its activated form, S-adenosylmethionine (SAM), as the principal one-carbon donor in the body. In the course of methyl group transfer from SAM, homocysteine (Hcy) is formed. Total plasma Hcy (tHcy) has been suggested as an independent risk factor for dementia and Alzheimer's disease [10-12]. There are a number of significant predictors of tHcy, such as serum folate [13], serum vitamin $B_{12}$ [14] and serum creatinine [15], but it is debated whether elevated tHcy is only a marker of or causally involved in the pathogenesis of cardiovascular disease and dementias [16].

A family history of dementia approximately doubles the lifetime risk of developing dementia [17]. Although the exact mechanisms are unknown, genetic, nutritional and environmental factors might contribute to the associations between dementia and tHcy, as many of these factors are common risk factors for both dementia and hyperhomocysteinemia. Thus, tHcy-related variables should also be studied in relation to family history of dementia.

Home care service (HCS) is intended to care for older people with and without dementia, to help them manage their own homes and to prevent premature institutionalization. As HCS in the majority of cases includes prefabricated food service ('meals on wheels'), it is of great importance to investigate how this food service influences nutrition in general and tHcy-related micronutrients in particular. Unfortunately, little attention has been paid to the risk of quantitative and qualitative malnutrition in the elderly [18]. However, the clinical utility of tHcy and its determinants in the laboratory work-up of patients with suspected dementia, as an adjunct to traditional patient history and demographic variables [19-21], is far from clear, and integrative studies in an out-patient based setting are required.

At the Memory Care Unit of the Department of Geriatrics, Örebro University Hospital, Sweden, a database was established in the mid-1990s to keep track of the patient records and to monitor the quality of the medical service concerning cognitive disorders. In the present study, we used this database to evaluate different patient history and demographic variables, as well as selected serum biomarkers as predictors of dementia in a consecutive series of 926 persons. The aim of the present study was to investigate potential differences in tHcy and its determinants [serum vitamin $B_{12}$, serum folate, serum creatinine and estimated glomerular filtration rate (eGFR)] in relation to the diagnosis of dementia. We also wanted to investigate associations between tHcy and vitamin $\mathrm{B}_{12}$ prescriptions, a family history of dementia as well as HCS requirements. 


\section{Materials and Methods}

\section{Study Population}

A database for monitoring the efficacy of various diagnostic markers and patient history data for differentiating between clinical dementia and non-dementia conditions in our Memory Care Unit was established in late 1995. The database comprises 926 consecutive subjects (366 men and 560 women) referred to the Memory Care Unit at the Geriatric Department, Örebro Medical Center Hospital, Örebro, Sweden, between 1996 and 2000 for diagnostic evaluation of suspected cognitive impairment. In this monocenter cross-sectional study, three specialists in geriatric medicine with comprehensive training in cognitive medicine performed a clinical investigation of these subjects. Dementia was diagnosed according to DSMIII-R criteria [22], which included medical history, physical examination, together with neurological, psychiatric and neuropsychological examination, and laboratory tests. At the time of patient recruitment, there was still no widespread use of multi-B vitamin supplements containing pharmacological vitamin $\mathrm{B}_{12}$ doses $(0.5 \mathrm{mg} /$ tablet $)$ through doctor's prescription in Sweden, whereas around $15 \%$ of subjects aged $\geq 70$ years were on high-dose oral ( $1 \mathrm{mg} /$ day $)$ or parenteral vitamin $\mathrm{B}_{12}$ treatment and approximately $3 \%$ were on folic acid treatment [23].

A wide range of biochemical blood tests and patient history variables was entered into the database. In the present study, we focus on the following variables: prescription of highdose oral or intermittent parenteral vitamin $B_{12}$ preparations (yes/no), family history of dementia (yes/no) and the use of HCS (yes/no). The data on vitamin $B_{12}$ prescriptions was based on patient and caregiver history and refers only to high-dose oral (1-mg vitamin $\mathrm{B}_{12}$ tablets) or parenteral preparations $(1 \mathrm{mg} / \mathrm{ml})$. The dosage interval of parenteral vitamin $\mathrm{B}_{12}$ doses varied from $1 \mathrm{mg}$ once a month to $1 \mathrm{mg}$ every third month. High-dose oral treatment accounted for approximately $70 \%$ of vitamin $\mathrm{B}_{12}$ prescriptions. In addition, we included the results from the Mini Mental State Examination (MMSE) tests in the analysis. Furthermore, data of serum biomarkers obtained by clinical routine testing at the time of recruitment [e.g. blood hemoglobin (B-Hb), blood mean cellular volume (B-MCV), serum albumin, plasma creatinine, serum folate, serum vitamin $B_{12}$, plasma tHcy, serum methylmalonic acid ( $\mathrm{S}$ MMA)] were retrieved from the database. In this paper, we focused on tHcy and variables related to $\mathrm{HCy}$ (serum folate, serum vitamin $\mathrm{B}_{12}$, S-MMA, plasma creatinine as well as B-Hb and B-MCV). eGFR was calculated according to the Modification of Diet in Renal Disease-4 formula (age, sex, ethnicity and plasma creatinine) [24]. Serum albumin and body mass index (BMI) were used as biomarkers of general nutritional status.

Because information about the use of folate supplementation was not recorded in the database, we used $35 \mathrm{nmol} / \mathrm{l}$ as a cutoff for serum folate to discriminate between subjects with and without folic acid supplementation. Subjects with a serum folate $>35 \mathrm{nmol} / \mathrm{l}$ were excluded from all statistical analyses related to tHcy. This cutoff has been shown to discriminate quite well between users and non-users of folic acid supplements [25]. In the dementia group, there were 7 subjects (1.6\%) with serum folate $>35 \mathrm{nmol} / \mathrm{l}$ and in the non-dementia group, there were 3 subjects $(1.8 \%)$.

\section{Biochemical Assays}

Venous blood samples were drawn with minimal venous stasis as a part of the routine dementia examination program between 8:00 and 10:00 a.m., and the samples were immediately delivered to the laboratory staff. The serum or plasma biomarker analyses were performed at the Department of Clinical Chemistry, Örebro Medical Center Hospital, Sweden, except for S-MMA which was analyzed by mass spectrometry at the Department of Clinical Chemistry, Central Hospital, Karlstad, Sweden. The local reference range of S-MMA is $<0.37 \mu \mathrm{mol} / \mathrm{l}$ (CV (coefficient of variance): $6.8 \%$ ) and the tHcy reference range is $<15 \mu \mathrm{mol} / \mathrm{l}(\mathrm{CV}: 4.1 \%)$. 
Table 1. Baseline characteristics of the 926 subjects studied

\begin{tabular}{lccc}
\hline & $\begin{array}{l}\text { Non-dementia } \\
(\mathrm{n}=240)\end{array}$ & $\begin{array}{l}\text { Dementia } \\
(\mathrm{n}=686)\end{array}$ & $\mathrm{p}^{*}$ \\
\hline Age, years & $77.9 \pm 9.6$ & $79.5 \pm 7.3$ & 0.010 \\
Female sex, $\mathrm{n} /$ total $\mathrm{n}(\%)$ & $144 / 240(60.0)$ & $416 / 686(60.6)$ & 0.163 \\
Positive family history of dementia, n/total $\mathrm{n}(\%)$ & $52 / 167(31.1)$ & $191 / 481(39.7)$ & 0.049 \\
B-Hb, g/l & $131.7 \pm 15.9$ & $130.8 \pm 15.5$ & 0.536 \\
MCV, fl & $90.0 \pm 5.8$ & $90.0 \pm 4.9$ & 0.899 \\
BMI & $24.0 \pm 4.3$ & $24.0 \pm 4.0$ & 0.997 \\
Serum albumin, g/l & $36.8 \pm 4.6$ & $36.6 \pm 4.6$ & 0.565 \\
Plasma creatinine, $\mu$ mol/l & $93.9 \pm 31.6$ & $94.1 \pm 46.7$ & 0.662 \\
eGFR, ml/min/1.73 m ${ }^{2}$ & $59.3 \pm 19.6$ & $59.9 \pm 18.9$ & 0.730 \\
Systolic BP, mm Hg & $149.8 \pm 25.4$ & $149.2 \pm 23.8$ & 0.783 \\
Diastolic BP, mm Hg & $79.5 \pm 12.5$ & $78.9 \pm 11.7$ & 0.544 \\
MMSE & $23.5 \pm 4.9$ & $16.3 \pm 6.1$ & $<0.001$ \\
Vitamin-B ${ }_{12} \mathrm{prescription}, \mathrm{n} /$ total $\mathrm{n}(\%)$ & $47 / 240(19.6)$ & $213 / 686(31.0)$ & 0.001 \\
Plasma tHcy, $\mu \mathrm{mol} / \mathrm{l}$ & $18.44 \pm 9.4$ & $20.6 \pm 9.1$ & 0.088 \\
S-MMA, $\mu \mathrm{mol} / \mathrm{l}$ & $0.23 \pm 0.15$ & $0.32 \pm 0.36$ & 0.176 \\
\hline
\end{tabular}

Data are means $\pm S D$, unless otherwise indicated. $\mathrm{BP}=$ Blood pressure. ${ }^{*}$ Pearson $\chi^{2}$ or $\mathrm{t}$ test as appropriate (calculated on logarithmically transformed variables).

\section{Statistical Analyses}

Statistical analyses were performed using SPSS for Windows, release 16 (SPSS Inc., Chicago, Ill., USA). None of the continuous variables were normally distributed according to Kolmogorov-Smirnov goodness-of-fit test and thus, analyses were performed on logarithmically transformed data. The descriptive results are presented as means \pm SD. Statistical significance was considered with a two-sided probability value $\leq 0.05$. Student's $t$ test was used for statistical analysis of differences between groups of continuous variables and $\chi^{2}$ tests were used for categorical variables. The patient history data were coded as binary variables (yes/ no). Associations between variables were assessed using Spearman rank correlations. Multivariate logistic regression analyses was carried out with the forward logistic regression method, with dementia/non-dementia being the dependent variable.

\section{Ethics}

The Regional Ethical Review Board, Uppsala, Sweden, approved the study.

\section{Results}

A total of 686 of the examined 926 subjects fulfilled the diagnostic criteria of dementia. The remaining 240 were categorized as non-demented. The baseline characteristics of the subjects are shown in table 1 . The subjects in the dementia group were marginally older than subjects in the non-dementia group. Demented subjects also more frequently had a positive family history of dementia and more often received high-dose oral or intermittent parenteral vitamin $B_{12}$ prescriptions than non-demented subjects. The MMSE was significantly lower in the dementia group $(\mathrm{p}<0.001)$ than in the non-dementia group. There were no sig- 
Table 2. Bivariate association between age and selected variables from table 1

\begin{tabular}{|c|c|c|c|c|c|c|c|c|c|c|}
\hline & & P-tHcy & S-MMA & Age & $\mathrm{B}-\mathrm{Hb}$ & B-MCV & S-Alb & P-Crea & BMI & eGFR \\
\hline \multirow[t]{3}{*}{ P-tHcy } & Correlation coefficient & 1.000 & 0.471 & 0.243 & -0.147 & 0.011 & -0.049 & 0.245 & -0.122 & -0.259 \\
\hline & Significance (two-tailed) & & 0.001 & $<0.001$ & 0.021 & 0.878 & 0.449 & $<0.001$ & 0.061 & $<0.001$ \\
\hline & $\mathrm{n}$ & 257 & 46 & 257 & 249 & 212 & 246 & 247 & 234 & 247 \\
\hline \multirow{3}{*}{\multicolumn{2}{|c|}{$\begin{array}{l}\text { S-MMA Correlation coefficient } \\
\text { Significance (two-tailed) } \\
n\end{array}$}} & 0.471 & 1.000 & 0.550 & -0.478 & 0.092 & -0.303 & 0.323 & 0.234 & -0.355 \\
\hline & & 0.001 & & $<0.001$ & $<0.001$ & 0.515 & 0.018 & 0.012 & 0.086 & 0.005 \\
\hline & & 46 & 61 & 61 & 60 & 52 & 60 & 60 & 55 & 60 \\
\hline \multirow[t]{3}{*}{ Age } & ficient & 0.243 & 0.550 & 1.000 & -0.253 & -0.060 & -0.272 & 0.164 & -0.159 & -0.225 \\
\hline & Significance (two-tailed) & $<0.001$ & $<0.001$ & & $<0.001$ & 0.162 & $<0.001$ & $<0.001$ & $<0.001$ & $<0.001$ \\
\hline & $\mathrm{n}$ & 257 & 61 & 926 & 753 & 544 & 704 & 728 & 704 & 728 \\
\hline \multirow[t]{3}{*}{$\mathrm{B}-\mathrm{Hb}$} & Correlation coefficient & -0.147 & -0.478 & -0.253 & 1.000 & 0.062 & 0.417 & -0.003 & 0.223 & 0.025 \\
\hline & Significance (two-tailed) & 0.021 & $<0.001$ & $<0.001$ & & 0.151 & $<0.001$ & 0.926 & $<0.001$ & 0.505 \\
\hline & $\mathrm{n}$ & 249 & 60 & 753 & 753 & 544 & 693 & 714 & 622 & 714 \\
\hline \multirow[t]{3}{*}{$\mathrm{B}-\mathrm{MCV}$} & Correlation coefficient & 0.011 & 0.092 & -0.060 & 0.062 & 1.000 & 0.046 & 0.048 & 0.029 & -0.044 \\
\hline & Significance (two-tailed) & 0.878 & 0.515 & 0.162 & 0.151 & & 0.295 & 0.272 & 0.526 & 0.318 \\
\hline & $\mathrm{n}$ & 212 & 52 & 544 & 544 & 753 & 522 & 523 & 491 & 523 \\
\hline \multirow[t]{3}{*}{ S-Alb } & Correlation coefficient & -0.049 & -0.303 & -0.272 & 0.417 & 0.046 & 1.000 & 0.064 & 0.163 & -0.043 \\
\hline & Significance (two-tailed) & 0.449 & 0.018 & $<0.001$ & $<0.001$ & 0.295 & & 0.093 & $<0.001$ & 0.259 \\
\hline & $\mathrm{n}$ & 246 & 60 & 704 & 693 & 522 & 704 & 687 & 592 & 687 \\
\hline \multirow[t]{3}{*}{ P-Crea } & Correlation coefficient & 0.245 & 0.323 & 0.164 & -0.003 & 0.048 & 0.064 & 1.000 & 0.166 & -0.997 \\
\hline & Significance (two-tailed) & $<0.001$ & 0.012 & $<0.001$ & 0.926 & 0.272 & 0.093 & & $<0.001$ & $<0.001$ \\
\hline & $\mathrm{n}$ & 247 & 60 & 728 & 714 & 523 & 687 & 728 & 610 & 728 \\
\hline \multirow[t]{3}{*}{$\mathrm{BMI}$} & Correlation coefficient & -0.122 & 0.234 & -0.159 & 0.223 & 0.029 & 0.163 & 0.166 & 1.000 & -0.153 \\
\hline & Significance (two-tailed) & 0.061 & 0.086 & $<0.001$ & $<0.001$ & 0.526 & $<0.001$ & $<0.001$ & & $<0.001$ \\
\hline & $\mathrm{n}$ & 234 & 55 & 704 & 622 & 491 & 592 & 610 & 703 & 610 \\
\hline \multirow[t]{3}{*}{ eGFR } & Correlation coefficient & -0.259 & -0.355 & -0.225 & 0.025 & -0.044 & -0.043 & -0.997 & -0.153 & 1.000 \\
\hline & Significance (two-tailed) & $<0.001$ & 0.005 & $<0.001$ & 0.505 & 0.318 & 0.259 & $<0.001$ & $<0.001$ & \\
\hline & $\mathrm{n}$ & 247 & 60 & 728 & 714 & 523 & 687 & 728 & 610 & 728 \\
\hline
\end{tabular}

$\mathrm{p}$ values according to nonparametric two-tailed Spearman correlation calculations. $\mathrm{P}-\mathrm{tHcy}=\mathrm{Plasma} \mathrm{tHcy}$; $\mathrm{S}-\mathrm{Alb}=\mathrm{se}-$ rum albumin; P-Crea = plasma creatinine.

nificant differences between dementia and non-dementia concerning B-Hb, B-MCV, BMI, serum albumin, eGFR, systolic or diastolic blood pressure.

Table 2 shows crude bivariate analysis (Spearman rank) of the associations of the selected biochemical parameters with age. Significant associations were seen between age and tHcy $(\mathrm{R}=0.243 ; \mathrm{p}<0.001)$, age and MMA $(\mathrm{R}=0.550 ; \mathrm{p}<0.001)$, age and $\mathrm{B}-\mathrm{Hb}(\mathrm{R}=-0.253$; $\mathrm{p}<0.001)$, age and serum albumin $(\mathrm{R}=-0.272 ; \mathrm{p}<0.001)$, age and plasma creatinine $(\mathrm{R}=$ $0.164 ; \mathrm{p}<0.001)$, age and eGFR $(\mathrm{R}=-0.225 ; \mathrm{p}<0.001)$, and age and $\mathrm{BMI}(\mathrm{R}=-0.159 ; \mathrm{p}<$ $0.001)$.

Table 3 shows the vitamin $\mathrm{B}_{12^{-}}$and folate-related variables (e.g. tHcy, S-MMA, B-Hb and $\mathrm{B}-\mathrm{MCV}$ ) in relation to presence or absence of vitamin $\mathrm{B}_{12}$ prescription. In the dementia group, there were 7 subjects $(1.8 \%)$ with serum folate $>35 \mathrm{nmol} / \mathrm{l}$ and in the non-dementia group, there were 3 subjects (1.8\%). These cases were all excluded from the statistical calcu- 
Table 3. Biochemical parameters according to vitamin $B_{12}$ prescription state and dementia diagnosis

\begin{tabular}{|c|c|c|c|c|c|c|c|c|}
\hline \multicolumn{2}{|l|}{ Variable } & \multicolumn{2}{|c|}{ Non-dementia $n$} & \multirow{3}{*}{$\frac{\mathrm{p}^{1}}{0.020}$} & \multirow{3}{*}{$\begin{array}{l}\text { Dementia } \\
334.0 \pm 325.5 \\
317.8 \pm 168.2 \\
\end{array}$} & \multirow{3}{*}{$\begin{array}{l}n \\
126 \\
231\end{array}$} & \multirow{3}{*}{$\frac{\mathrm{p}^{1}}{0.025}$} & \multirow{3}{*}{$\begin{array}{l}p^{2} \\
0.654 \\
0.340\end{array}$} \\
\hline $\mathrm{S}-\mathrm{B}_{12}, \mathrm{pmol} / \mathrm{l}$ & $+\mathrm{B}_{12}$ & $292.8 \pm 250.4$ & 24 & & & & & \\
\hline & $-\mathrm{B}_{12}$ & $349.8 \pm 233.2$ & 100 & & & & & \\
\hline \multirow[t]{2}{*}{ S-folate, nmol/l } & $+\mathrm{B}_{12}$ & $8.6 \pm 3.4$ & 39 & \multirow[t]{2}{*}{0.006} & $8.3 \pm 4.0$ & 153 & \multirow[t]{2}{*}{$<0.001$} & 0.454 \\
\hline & $-\mathrm{B}_{12}$ & $11.6 \pm 6.5$ & 121 & & $10.7 \pm 5.3$ & 265 & & 0.351 \\
\hline \multirow[t]{2}{*}{ P-tHcy, $\mu \mathrm{mol} / \mathrm{l}$} & $+\mathrm{B}_{12}$ & $22.7 \pm 11.5$ & 29 & \multirow[t]{2}{*}{0.001} & $24.0 \pm 9.0$ & 77 & \multirow[t]{2}{*}{$<0.001$} & 0.267 \\
\hline & $-\mathrm{B}_{12}$ & $15.9 \pm 5.8$ & 43 & & $17.6 \pm 8.3$ & 79 & & 0.389 \\
\hline \multirow[t]{2}{*}{ S-MMA, $\mu \mathrm{mol} / 1$} & $+\mathrm{B}_{12}$ & $0.38 \pm 0.21$ & 5 & \multirow[t]{2}{*}{0.023} & $0.46 \pm 0.50$ & 17 & \multirow[t]{2}{*}{0.032} & 0.885 \\
\hline & $-\mathrm{B}_{12}$ & $0.19 \pm 0.10$ & 13 & & $0.24 \pm 0.16$ & 15 & & 0.254 \\
\hline \multirow[t]{2}{*}{$\mathrm{B}-\mathrm{Hb}, \mathrm{g} / \mathrm{l}$} & $+\mathrm{B}_{12}$ & $125.7 \pm 17.6$ & 36 & \multirow[t]{2}{*}{0.004} & $127.7 \pm 14.9$ & 150 & \multirow[t]{2}{*}{0.004} & 0.400 \\
\hline & $-\mathrm{B}_{12}$ & $134.3 \pm 15.0$ & 120 & & $132.3 \pm 15.4$ & 249 & & 0.242 \\
\hline \multirow[t]{2}{*}{$\mathrm{B}-\mathrm{MCV}, \mathrm{fl}$} & $+\mathrm{B}_{12}$ & $88.0 \pm 6.4$ & 27 & \multirow[t]{2}{*}{0.015} & $90.8 \pm 4.8$ & 123 & \multirow[t]{2}{*}{0.099} & 0.011 \\
\hline & $-\mathrm{B}_{12}$ & $90.8 \pm 4.9$ & 92 & & $89.8 \pm 4.8$ & 186 & & 0.137 \\
\hline \multirow[t]{2}{*}{ S-Alb, g/l } & $+\mathrm{B}_{12}$ & $36.1 \pm 5.3$ & 35 & \multirow[t]{2}{*}{0.167} & $35.4 \pm 4.2$ & 146 & \multirow[t]{2}{*}{0.001} & 0.534 \\
\hline & $-\mathrm{B}_{12}$ & $37.2 \pm 4.5$ & 117 & & $36.9 \pm 4.4$ & 242 & & 0.537 \\
\hline \multirow[t]{2}{*}{ P-Crea, $\mu \mathrm{mol} / 1$} & $+\mathrm{B}_{12}$ & $91.2 \pm 23.5$ & 38 & \multirow[t]{2}{*}{0.874} & $89.1 \pm 30.4$ & 146 & \multirow[t]{2}{*}{0.274} & 0.458 \\
\hline & $-\mathrm{B}_{12}$ & $94.2 \pm 34.0$ & 119 & & $91.8 \pm 29.2$ & 248 & & 0.668 \\
\hline \multirow{2}{*}{$\begin{array}{l}\text { eGFR } \\
\mathrm{ml} / \mathrm{min} / 1.73 \mathrm{~m}^{2}\end{array}$} & $+\mathrm{B}_{12}$ & $59.1 \pm 15.3$ & 38 & \multirow[t]{2}{*}{0.710} & $60.3 \pm 19.7$ & 146 & \multirow[t]{2}{*}{0.561} & 0.707 \\
\hline & $-\mathrm{B}_{12}$ & $59.2 \pm 20.7$ & 119 & & $59.6 \pm 18.4$ & 242 & & 0.847 \\
\hline \multirow[t]{2}{*}{ BMI } & $+\mathrm{B}_{12}$ & $23.5 \pm 3.9$ & 35 & \multirow[t]{2}{*}{0.404} & $23.4 \pm 3.7$ & 133 & \multirow[t]{2}{*}{0.126} & 0.867 \\
\hline & $-\mathrm{B}_{12}$ & $24.2 \pm 4.3$ & 106 & & $24.1 \pm 4.2$ & 223 & & 0.799 \\
\hline
\end{tabular}

Values are means \pm SD. $p$ values are calculated by $t$ test on logarithmically transformed variables. $\mathrm{S}-\mathrm{B}_{12}=$ Serum vitamin $\mathrm{B}_{12} ; \mathrm{P}$-tHcy = plasma tHcy; $\mathrm{S}-\mathrm{Alb}=$ serum albumin; $\mathrm{P}-\mathrm{Crea}=$ plasma creatinine; $+\mathrm{B}_{12}=$ has been prescribed vitamin $\mathrm{B}_{12} ;-\mathrm{B}_{12}=$ has not been prescribed vitamin $\mathrm{B}_{12} .{ }^{1}$ Difference between the $+B_{12}$ prescription and $-B_{12}$ prescription groups. ${ }^{2}$ Difference between the non-dementia and dementia groups.

lations. Both dementia and non-dementia subjects with a vitamin $\mathrm{B}_{12}$ prescription had significantly higher tHcy levels ( $\mathrm{p}=0.001$ and $<0.001$, respectively) but lower serum folate (non-dementia: $\mathrm{p}<0.001$; dementia: $\mathrm{p}=0.006$ ). S-MMA was also significantly higher in subjects with vitamin $B_{12}$ prescription, both in the dementia and the non-dementia groups $\left(\mathrm{p}=0.032\right.$ and 0.023 , respectively). B-Hb was significantly lower in vitamin $B_{12}$-prescribed dementia $(\mathrm{p}=0.004)$ and non-dementia $(\mathrm{p}=0.004)$ subjects than in subjects not prescribed vitamin $\mathrm{B}_{12}$.

Table 4 shows the tHcy-related variables in relation to family history of dementia. Among subjects with a positive family history of dementia, mean tHcy was significantly higher in demented subjects than in non-demented subjects $(\mathrm{p}=0.021)$. Non-demented subjects with a positive family history of dementia had significantly higher eGFR than non-demented subjects without a family history of dementia $(\mathrm{p}=0.032)$.

Table 5 depicts differences in biomarkers in non-demented and demented subjects according to HCS state. There were no significant differences in serum vitamin $\mathrm{B}_{12}$ or in serum folate between the dementia and the non-dementia groups. The mean tHcy level was sig- 
Table 4. Hcy-related biomarkers in relation to family history of dementia and data completeness ( $t$ test statistics)

\begin{tabular}{|c|c|c|c|c|c|c|c|c|}
\hline \multicolumn{2}{|l|}{ Variable } & \multirow{2}{*}{$\begin{array}{l}\text { Non-dementia } \\
303.8 \pm 137.1\end{array}$} & \multirow{2}{*}{$\frac{n}{31}$} & \multirow{3}{*}{$\frac{\mathrm{p}^{1}}{0.912}$} & \multirow{3}{*}{$\begin{array}{l}\text { Dementia } \\
272.4 \pm 121.0 \\
340.8 \pm 265.0\end{array}$} & \multirow{3}{*}{$\begin{array}{l}\mathrm{n} \\
107 \\
147\end{array}$} & \multirow{3}{*}{$\frac{\mathrm{p}^{1}}{0.136}$} & \multirow{3}{*}{$\begin{array}{l}\mathrm{p}^{2} \\
0.253 \\
0.917\end{array}$} \\
\hline$S-B_{12}, \mathrm{pmol} / \mathrm{l}$ & $+\mathrm{FH}$ & & & & & & & \\
\hline & $-\mathrm{FH}$ & $330.0 \pm 247.2$ & 60 & & & & & \\
\hline \multirow{2}{*}{ S-folate, $\mathrm{nmol} / \mathrm{l}$} & $+\mathrm{FH}$ & $12.4 \pm 7.7$ & 36 & 0.521 & $10.8 \pm 5.7$ & 120 & 0.236 & 0.225 \\
\hline & $-\mathrm{FH}$ & $11.3 \pm 5.7$ & 76 & & $10.1 \pm 5.1$ & 167 & & 0.087 \\
\hline \multirow[t]{2}{*}{ P-tHcy, $\mu \mathrm{mol} / \mathrm{l}$} & $+\mathrm{FH}$ & $15.1 \pm 3.9$ & 13 & 0.168 & $20.6 \pm 9.4$ & 37 & 0.744 & 0.021 \\
\hline & $-\mathrm{FH}$ & $18.8 \pm 8.9$ & 35 & & $20.2 \pm 6.9$ & 59 & & 0.191 \\
\hline \multirow{2}{*}{ S-MMA, $\mu \mathrm{mol} / \mathrm{l}$} & $+\mathrm{FH}$ & $0.19 \pm 0.13$ & 7 & 0.431 & $0.48 \pm 0.74$ & 8 & 0.605 & 0.219 \\
\hline & $-\mathrm{FH}$ & $0.26 \pm 0.20$ & 6 & & $0.27 \pm 0.17$ & 13 & & 0.736 \\
\hline \multirow{2}{*}{ B-Hb, g/l } & $+\mathrm{FH}$ & $133.7 \pm 14.3$ & 34 & 0.615 & $133.1 \pm 12.9$ & 114 & 0.183 & 0.980 \\
\hline & $-\mathrm{FH}$ & $135.0 \pm 15.6$ & 75 & & $131.1 \pm 15.5$ & 159 & & 0.081 \\
\hline \multirow[t]{2}{*}{$\mathrm{B}-\mathrm{MCV}, \mathrm{fl}$} & $+\mathrm{FH}$ & $90.8 \pm 5.9$ & 27 & 0.444 & $89.4 \pm 5.1$ & 87 & 0.074 & 0.252 \\
\hline & $-\mathrm{FH}$ & $89.8 \pm 5.2$ & 58 & & $90.6 \pm 4.5$ & 125 & & 0.284 \\
\hline \multirow[t]{2}{*}{ S-Alb, g/l } & $+\mathrm{FH}$ & $38.2 \pm 4.3$ & 35 & 0.521 & $37.9 \pm 3.4$ & 111 & $<0.001$ & 0.842 \\
\hline & $-\mathrm{FH}$ & $37.6 \pm 4.5$ & 72 & & $36.2 \pm 4.3$ & 151 & & 0.024 \\
\hline \multirow[t]{2}{*}{ P-Crea, $\mu \mathrm{mol} / \mathrm{l}$} & $+\mathrm{FH}$ & $85.6 \pm 22.7$ & 45 & 0.047 & $88.1 \pm 24.6$ & 148 & 0.312 & 0.517 \\
\hline & $-\mathrm{FH}$ & $96.0 \pm 30.2$ & 90 & & $95.6 \pm 61.6$ & 222 & & 0.323 \\
\hline \multirow{2}{*}{$\begin{array}{l}\text { eGFR } \\
\mathrm{ml} / \mathrm{min} / 1.73 \mathrm{~m}^{2}\end{array}$} & $+\mathrm{FH}$ & $64.0 \pm 17.2$ & 45 & 0.032 & $61.5 \pm 16.3$ & 148 & 0.277 & 0.425 \\
\hline & $-\mathrm{FH}$ & $57.7 \pm 19.2$ & 90 & & $60.9 \pm 20.3$ & 222 & & 0.323 \\
\hline \multirow[t]{2}{*}{ BMI } & $+\mathrm{FH}$ & $24.1 \pm 4.6$ & 34 & 0.867 & $24.6 \pm 3.8$ & 109 & 0.024 & 0.438 \\
\hline & $-\mathrm{FH}$ & $24.3 \pm 4.6$ & 70 & & $23.5 \pm 4.1$ & 145 & & 0.252 \\
\hline
\end{tabular}

Values are means $\pm \mathrm{SD}$. $\mathrm{p}$ values are calculated by $\mathrm{t}$ test on logarithmically transformed variables. $\mathrm{S}-\mathrm{B}_{12}=$ Serum vitamin $\mathrm{B}_{12} ; \mathrm{P}-\mathrm{tHcy}=$ plasma tHcy; $\mathrm{S}-\mathrm{Alb}=$ serum albumin; $\mathrm{P}-\mathrm{Crea}=$ plasma creatinine; $+\mathrm{FH}=$ positive family history of dementia; $-\mathrm{FH}=$ negative family history of dementia. ${ }^{1}$ Difference between the $+\mathrm{FH}$ and $-\mathrm{FH}$ groups. ${ }^{2}$ Difference between the non-dementia and dementia groups.

nificantly higher in the non-dementia group with HCS $(\mathrm{p}=0.007)$ and borderline significantly higher in the dementia group with HCS $(\mathrm{p}=0.064)$. Likewise, mean S-MMA was higher in the non-demented subgroup with HCS $(\mathrm{p}=0.021)$ and tended to be higher in the demented subgroup with HCS $(\mathrm{p}=0.140)$. Furthermore, there was a significant difference in eGFR between demented and non-demented subjects receiving HCS. Non-demented subjects with HCS had lower eGFR than demented subjects with HCS $(\mathrm{p}=0.034)$. Subjects receiving HCS had both significantly lower B-Hb (dementia: $\mathrm{p}<0.001$; non-dementia: $\mathrm{p}=$ 0.037 ) and serum albumin (dementia: $\mathrm{p}<0.001$; non-dementia: $\mathrm{p}=0.004$ ) compared with subjects without HCS. BMI was found to be significantly lower in subjects in the dementia group receiving HCS compared to demented subjects without HCS $(\mathrm{p}=0.05)$. This difference was not seen in non-demented subjects with and without HCS.

Table 6 shows the odds ratio of having a diagnosis of dementia in relation to the three patient history variables as calculated by multivariate logistic regression analysis. Use of HCS, a family history of dementia and prescription of vitamin $B_{12}$ preparations were positively associated with increased risk of dementia. 
Table 5. Biochemical parameters according to the HCS state and dementia diagnosis

\begin{tabular}{|c|c|c|c|c|c|c|c|c|}
\hline Variable & & Non-dementia & $\mathrm{n}$ & $\mathrm{p}^{1}$ & Dementia & $\mathrm{n}$ & $\mathrm{p}^{1}$ & $\mathrm{p}^{2}$ \\
\hline \multirow{2}{*}{$\mathrm{S}-\mathrm{B}_{12}, \mathrm{pmol} / \mathrm{l}$} & $+\mathrm{HCS}$ & $314.0 \pm 177.5$ & 45 & 0.521 & $317.3 \pm 197.7$ & 164 & 0.754 & 0.998 \\
\hline & $-\mathrm{HCS}$ & $353.9 \pm 266.2$ & 78 & & $326.9 \pm 261.8$ & 182 & & 0.280 \\
\hline \multirow[t]{2}{*}{ S-folate, nmol/l } & $+\mathrm{HCS}$ & $10.1 \pm 5.9$ & 60 & 0.137 & $9.3 \pm 4.7$ & 195 & 0.064 & 0.461 \\
\hline & $-\mathrm{HCS}$ & $11.3 \pm 6.1$ & 99 & & $10.3 \pm 5.3$ & 211 & & 0.167 \\
\hline \multirow[t]{2}{*}{ P-tHcy, $\mu \mathrm{mol} / 1$} & $+\mathrm{HCS}$ & $22.4 \pm 12.0$ & 28 & 0.007 & $21.4 \pm 7.9$ & 73 & 0.064 & 0.942 \\
\hline & $-\mathrm{HCS}$ & $16.3 \pm 5.6$ & 44 & & $20.1 \pm 10.3$ & 80 & & 0.050 \\
\hline \multirow[t]{2}{*}{ S-MMA, $\mu \mathrm{mol} / \mathrm{l}$} & $+\mathrm{HCS}$ & $0.40 \pm 0.21$ & 4 & 0.021 & $0.49 \pm 0.59$ & 12 & 0.140 & 0.929 \\
\hline & $-\mathrm{HCS}$ & $0.20 \pm 0.11$ & 14 & & $0.28 \pm 0.18$ & 20 & & 0.090 \\
\hline \multirow[t]{2}{*}{ B-Hb, g/l } & $+\mathrm{HCS}$ & $129.0 \pm 17.3$ & 60 & 0.037 & $127.4 \pm 13.8$ & 198 & $<0.001$ & 0.602 \\
\hline & $-\mathrm{HCS}$ & $134.4 \pm 14.8$ & 95 & & $133.5 \pm 15.8$ & 198 & & 0.624 \\
\hline \multirow[t]{2}{*}{$\mathrm{B}-\mathrm{MCV}, \mathrm{fl}$} & $+\mathrm{HCS}$ & $90.6 \pm 6.0$ & 45 & 0.537 & $90.3 \pm 4.8$ & 147 & 0.703 & 0.765 \\
\hline & $-\mathrm{HCS}$ & $89.9 \pm 5.0$ & 73 & & $90.1 \pm 4.8$ & 153 & & 0.830 \\
\hline \multirow[t]{2}{*}{ S-Alb, g/l } & $+\mathrm{HCS}$ & $35.6 \pm 4.9$ & 57 & 0.004 & $35.5 \pm 4.3$ & 186 & $<0.001$ & 0.993 \\
\hline & $-\mathrm{HCS}$ & $37.8 \pm 4.5$ & 94 & & $37.2 \pm 4.3$ & 191 & & 0.328 \\
\hline \multirow[t]{2}{*}{ P-Crea, $\mu \mathrm{mol} / 1$} & $+\mathrm{HCS}$ & $98.5 \pm 35.2$ & 74 & 0.153 & $89.0 \pm 26.2$ & 257 & 0.016 & 0.517 \\
\hline & $-\mathrm{HCS}$ & $91.3 \pm 28.9$ & 119 & & $99.1 \pm 60.7$ & 263 & & 0.957 \\
\hline eGFR & $+\mathrm{HCS}$ & $56.4 \pm 20.1$ & 74 & 0.096 & $61.5 \pm 19.1$ & 257 & 0.037 & 0.034 \\
\hline $\mathrm{ml} / \mathrm{min} / 1.73 \mathrm{~m}^{2}$ & $-\mathrm{HCS}$ & $60.7 \pm 18.8$ & 119 & & $58.5 \pm 18.9$ & 263 & & 0.180 \\
\hline \multirow[t]{2}{*}{ BMI } & $+\mathrm{HCS}$ & $23.5 \pm 4.5$ & 48 & 0.180 & $23.4 \pm 4.0$ & 161 & 0.050 & 0.988 \\
\hline & $-\mathrm{HCS}$ & $24.4 \pm 4.1$ & 92 & & $24.2 \pm 4.0$ & 185 & & 0.791 \\
\hline
\end{tabular}

Values are means \pm SD. $p$ values are calculated by $t$ test on logarithmically transformed variables. $\mathrm{S}-\mathrm{B}_{12}=$ Serum vitamin $\mathrm{B}_{12} ; \mathrm{P}$-tHcy = plasma tHcy; $\mathrm{S}-\mathrm{Alb}=$ serum albumin; $\mathrm{P}-\mathrm{Crea}=$ plasma creatinine; $+\mathrm{HCS}=$ has HCS; $-\mathrm{HCS}=$ has not HCS. ${ }^{1}$ Difference between the $+\mathrm{HCS}$ and $-\mathrm{HCS}$ groups. ${ }^{2}$ Difference between the non-dementia and dementia groups.

Table 6. Odds ratio for dementia associated with different conditions

\begin{tabular}{lll}
\hline Condition (yes/no) & OR for dementia (95\% CI) & $\mathrm{p}$ \\
\hline Prescription of vitamin $\mathrm{B}_{12}$ (yes) & $2.01(1.29-3.13)$ & 0.002 \\
Family history of dementia (yes) & $1.50(1.02-2.19)$ & 0.032 \\
HCS (yes) & $1.47(1.01-2.13)$ & 0.044 \\
\hline
\end{tabular}

\section{Discussion}

Demented subjects more often received vitamin $\mathrm{B}_{12}$ supplementation than did the nondemented subjects. Despite high-dose oral or intermittent parenteral vitamin $\mathrm{B}_{12}$ supplementation, demented subjects still had significantly higher tHcy values and lower B-Hb and serum albumin than non-supplemented demented subjects. At the same time, there were no significant differences in B-MCV, BMI or eGFR. 
Hcy is regarded as an independent risk marker of dementia and Alzheimer's disease [10-12]. The association between vitamin $B_{12}$ and folate deficiency and dementia has been observed even in subjects without hematological signs of vitamin B deficiency [26]. Unfortunately, tHcy concentrations in addition to vitamin B deficiency may be influenced by a large number of genetic, physiological and lifestyle factors, impaired renal function being one of the most important confounders. Proper assessment of renal function is thus crucial. In addition to plasma creatinine, we calculated eGFR, which is considered a more precise marker of renal function.

Prior to the visit to our Memory Care Unit, clinicians had recognized the need for vitamin $B_{12}$ prescriptions in a significant portion of the studied subjects. About $30 \%$ in the dementia group and about $20 \%$ in the non-dementia group were supplemented with vitamin $\mathrm{B}_{12}$ at the time of the first visit at the Memory Care Unit. It is reasonable to assume that vitamin $B_{12}$ was originally prescribed because of low serum vitamin $B_{12}$ concentrations, but we were not able to retrieve data on how long the subjects had been prescribed vitamin $B_{12}$ before attending the Memory Care Unit. In the present material, there were no differences in serum vitamin $B_{12}$ concentrations between subjects with or without dementia. A paradox finding was that subjects with vitamin $B_{12}$ prescription actually had higher levels of both plasma tHcy and S-MMA (table 3). The reason for this is obscure, but could be related to a generally inferior health status and frailty among subjects receiving vitamin $B_{12}$ prescriptions. Those elderly receiving HCS had a poorer nutritional status and lower muscle mass. This could result in a misinterpretation of plasma creatinine values, being lower than they should be in relation to renal function. However, renal function as judged by eGFR did not differ between the groups in our material, so this is probably not an explanation for the higher plasma tHcy and S-MMA concentrations among patients receiving vitamin $\mathrm{B}_{12}$ supplementation. Other explanations could be that the prescribed vitamin $\mathrm{B}_{12}$ doses or dosage intervals were insufficient to meet the demands of the patients, that bioavailability of the given vitamin $B_{12}$ preparation was too low, the duration of treatment too short, that compliance especially in the demented group - was poor, or that many subjects suffering from vitamin $\mathrm{B}_{12}$ deficiency simultaneously had unrecognized folate deficiency before starting with vitamin $B_{12}$ supplementation. Oral vitamin $B_{12}$ administration, which in Sweden nowadays is the prevailing way of supplementation [27], has an inherent risk of poor compliance. Compared to intermittent parenteral vitamin $\mathrm{B}_{12}$ therapy, high-dose $(1 \mathrm{mg} /$ day) oral preparations have been demonstrated to be equally effective for maintenance therapy and even sufficient for the treatment of pernicious anemia [28]. The vitamin $\mathrm{B}_{12}$ doses in some oral multivitamin prescription preparations might, however, have been too low to cover the needs of all patient groups [29]. At the time when the study material was collected, there was no widespread use of multivitamin prescription preparations in Sweden. In our material, we only registered high-dose oral or intermittent injectable preparations of vitamin $\mathrm{B}_{12}$, the by far prevailing treatment option. The notion that insufficient treatment (too low doses, too short duration, too large dosage intervals and poor compliance) might be responsible for the paradoxical finding of higher tHcy and S-MMA values among subjects on vitamin $\mathrm{B}_{12}$ treatment is corroborated by the observation of relatively small differences in serum vitamin $\mathrm{B}_{12}$ concentrations between the supplemented and non-supplemented groups in our material (dementia: $\mathrm{p}=0.025$; non-dementia: $\mathrm{p}=0.020$ ). These factors, in addition to a general poor nutritional and health status, could explain higher MMA and tHcy levels in subjects with vitamin $B_{12}$ prescriptions in our material.

HCS is obviously not a guaranty of maintaining adequate nutritional status of the enrolled elderly subjects (table 5). Under- and malnutrition is a neglected problem even among institutionalized geriatric patients in Sweden [30]. This is reflected in our material too, since tHcy was generally higher in subjects assigned to HCS. This difference was most pronounced 
among the non-demented subjects $(\mathrm{p}=0.007)$, but we also observed a higher tHcy level in the dementia group receiving HCS, though it did not reach statistical significance $(\mathrm{p}=0.064)$. Two Finnish studies found that the majority of elderly Finnish home care patients were undernourished according to Mini Nutritional Assessment [31, 32]. BMI and serum albumin are useful biomarkers of the general nutritional status, especially among the elderly $[33,34]$. The finding that both demented and non-demented subjects with HCS had significantly lower serum albumin than the subjects without supports the notion that HCS in our material does not provide adequate nutrition. An alternative explanation could of course be 'confounding-by-indication'. Indeed, BMI was also lower among patients receiving HCS, but only in the demented group $(\mathrm{p}=0.050)$. The lower BMI may primarily be related to dementia itself and, as a result of this, increased requirement of HCS, rather than being caused by poorer general nutritional status as a consequence of HCS [35]. Further, HCS was a significant predisposing factor of dementia (odds ratio, OR 1.50, range 1.02-2.19), a finding most likely representing a reversed causality phenomenon. On the other hand, 'meals on wheels' combined with microwave heating, which is often provided by HCS, may negatively affect the vitamin B content of food [36].

HCS is in Sweden most often provided by the local community, and most of the expenditures for this service are paid by taxes. This service is intended to meet the requirements of free-living elderly and to allow them to stay self-supplied as long as possible and thereby postpone premature institutionalization. Sufficient nutrition must be one of the goals of HCS. Our findings of low serum albumin, elevated Hcy, high MMA, and low serum folate indicate that there are shortcomings in the HCS with regard to provision of an adequate nutritional status among the elderly in the studied group. Hcy has been proposed as a suitable biomarker for malnutrition [37-39].

We hypothesized that the group with a positive family history of dementia, which could indicate involvement of inherited factors in the development of dementia, would present changes in Hcy-related biomarkers. Indeed, we found a higher tHcy concentration in demented subjects with a positive family history of dementia (table 4). A possible explanation might be malfunction in the folate metabolism due to genetic polymorphisms, e.g. carrier state of the common methylenetetrahydrofolate reductase (MTHFR) 677C $>\mathrm{T}$ polymorphism [40]. Another possibility that we have looked into previously could be mutations in folate receptor- $\alpha[41,42]$.

High tHcy has previously been proposed to be an independent risk factor for dementia $[11,12,43,44]$. Our findings support a potential connection between high tHcy levels and dementia and that this association might be partly related to inherited factors and partly to environmental factors, such as poor nutrition. A positive family history of dementia increased the OR of having a dementia diagnosis by a factor of 1.5. This is a slightly lower OR compared to earlier epidemiological findings in a Swedish population-based sample, where the relative risk for subjects with a positive family history of dementia was 3.2 [17].

Perhaps the most likely explanation of the higher levels of tHcy concentrations in subjects prescribed vitamin $B_{12}$ is that supplementation with vitamin $B_{12}$ may induce a relative intracellular folate deficiency due to increased intracellular demands of folate. Increase in tHcy after mono-supplementation with vitamin $\mathrm{B}_{12}$ has been reported earlier [45]. Consistent with this 'folate stress' hypothesis, both dementia and non-dementia subjects with vitamin $B_{12}$ prescription also had significantly lower serum levels of folate. However, this hypothesis does not explain the elevated MMA concentrations in the same patient group.

A limitation of this study is that the database does not include information about the prescription of folic acid or the duration of and compliance with vitamin $\mathrm{B}_{12}$ supplementation, but we know that in 1995-2000, when the present study population was recruited, the use of multi-B vitamin prescriptions containing lower vitamin $B_{12}$ doses than vitamin $B_{12}$ 
mono-tablets ( 0.5 vs. $1 \mathrm{mg} /$ tablet $)$ in Sweden was not widespread, and we used a serum folate cutoff level of $35 \mathrm{nmol} / \mathrm{l}$ as an exclusion criterion, which has been proposed to discriminate quite well between those taking folic acid supplements (including subjects taking over-thecounter multi-B vitamins) and those who do not [25]. Another restraint is that the data set was not complete with regard to medical history information and blood biomarkers in all subjects. While medical history information could be retrieved in almost all cases, information on biochemical parameters was in part incomplete.

In summary, we observed that tHcy levels were higher among subjects who were supplemented with vitamin $\mathrm{B}_{12}$. This finding could not be explained by poorer renal function among these subjects. Further, we identified a positive family history of dementia and the need for HCS as contributing factors to this paradoxical finding. Thus, regular monitoring of the nutritional status in elderly geriatric patients is necessary. A broader approach to improve nutrition especially among demented elderly is warranted [46]. HCS providers might need to pay greater attention to nutrition and compliance in demented and non-demented subjects. We advocate a more active use of biomarkers as an aid to earlier identify subjects being at risk of nutritional insufficiency and to improve quality of health care among freeliving as well as institutionalized elderly.

\section{Acknowledgements}

This study was supported by grants from the Örebro County Council, Örebro University, and Nyckelfonden. We thank Dr. Lars H. Breimer for his valuable comments on the manuscript.

\section{Disclosure Statement}

None of the authors declare any conflict of interest.

\section{References}

1 Addison T: Anemia: disease of the suprarenal capsules. London Med Gaz 1849;43:517-518.

-2 van Asselt DZ, Pasman JW, van Lier HJ, Vingerhoets DM, Poels PJ, Kuin Y, Blom HJ, Hoefnagels WH: Cobalamin supplementation improves cognitive and cerebral function in older, cobalamindeficient persons. J Gerontol A Biol Sci Med Sci 2001;56:M775-M779.

-3 Nilsson K, Warkentin S, Hultberg B, Fäldt R, Gustafson L: Treatment of cobalamin deficiency in dementia, evaluated clinically and with cerebral blood flow measurements. Aging Clin Exp Res 2000; 12:199-207.

-4 Strachan RW, Henderson JG: Dementia and folate deficiency. Q J Med 1967;36:189-204.

5 Sapira JD, Tullis S, Mullaly R: Reversible dementia due to folate deficiency. South Med J 1975;68: 776-777.

6 Martin DC: B12 and folate deficiency dementia. Clin Geriatr Med 1988;4:841-852.

-7 Mischoulon D, Raab MF: The role of folate in depression and dementia. J Clin Psychiatry 2007; 68(suppl 10):28-33.

-8 Tchantchou F, Shea TB: Folate deprivation, the methionine cycle, and Alzheimer's disease. Vitam Horm 2008;79:83-97.

-9 Maxwell CJ, Hogan DB, Ebly EM: Serum folate levels and subsequent adverse cerebrovascular outcomes in elderly persons. Dement Geriatr Cogn Disord 2002;13:225-234. 
10 McCaddon A, Davies G, Hudson P, Tandy S, Cattell H: Total serum homocysteine in senile dementia of Alzheimer type. Int J Geriatr Psychiatry 1998;13:235-239.

-11 Seshadri S, Beiser A, Selhub J, Jacques PF, Rosenberg IH, D’Agostino RB, Wilson PW, Wolf PA: Plasma homocysteine as a risk factor for dementia and Alzheimer's disease. N Engl J Med 2002;346: 476-483.

$\checkmark 12$ Smith AD: The worldwide challenge of the dementias: a role for B vitamins and homocysteine? Food Nutr Bull 2008;29:S143-S172.

-13 Lee BJ, Lin PT, Liaw YP, Chang SJ, Cheng CH, Huang YC: Homocysteine and risk of coronary artery disease: Folate is the important determinant of plasma homocysteine concentration. Nutrition 2003; 19:577-583.

-14 Robertson J, Iemolo F, Stabler SP, Allen RH, Spence JD: Vitamin B12, homocysteine and carotid plaque in the era of folic acid fortification of enriched cereal grain products. CMAJ 2005;172:15691573.

-15 Lin YH, Pao KY, Wu VC, Lin YL, Chien YF, Hung CS, Chen YJ, Liu CP, Tsai IJ, Gau CS, Wu KD, Hwang JJ: The influence of estimated creatinine clearance on plasma homocysteine in hypertensive patients with normal serum creatinine. Clin Biochem 2007;40:230-234.

-16 Brattström L, Wilcken DE: Homocysteine and cardiovascular disease: cause or effect? Am J Clin Nutr 2000;72:315-323.

-17 Fratiglioni L, Ahlbom A, Viitanen M, Winblad B: Risk factors for late-onset Alzheimer's disease: a population-based, case-control study. Ann Neurol 1993;33:258-266.

18 Johansson L, Sidenvall B, Malmberg B, Christensson L: Who will become malnourished? A prospective study of factors associated with malnutrition in older persons living at home. J Nutr Health Aging 2009;13:855-861.

19 Wallin A, Brun A, Gustafson L: Swedish consensus on dementia diseases. Acta Neurol Scand Suppl 1994;90:1-31.

-20 Waldemar G, Dubois B, Emre M, Scheltens P, Tariska P, Rossor M: Diagnosis and management of Alzheimer's disease and other disorders associated with dementia. The role of neurologists in Europe. European Federation of Neurological Societies. Eur J Neurol 2000;7:133-144.

-21 Atti AR, Palmer K, Volpato S, Winblad B, De Ronchi D, Fratiglioni L: Late-life body mass index and dementia incidence: nine-year follow-up data from the Kungsholmen Project. J Am Geriatr Soc 2008; 56:111-116.

22 Diagnostic and Statistical Manual of Mental Disorders, ed 3. Revised (DSM-III-R). Washington, DC, American Psychiatric Association, 1987.

-23 Björkegren K, Svärdsudd K: Elevated serum levels of methylmalonic acid and homocysteine in elderly people. A population-based intervention study. J Intern Med 1999;246:317-324.

24 Levey A, Green T, Kusek J, Beck G: A simplified equation to predict glomerular filtration rate from serum creatinine. J Am Soc Nephrol 2000;11, Abstract

-25 Wolters M, Hermann S, Hahn A: Effect of multivitamin supplementation on the homocysteine and methylmalonic acid blood concentrations in women over the age of 60 years. Eur J Nutr 2005;44: 183-192.

-26 Lindenbaum J, Healton EB, Savage DG, Brust JC, Garrett TJ, Podell ER, Marcell PD, Stabler SP, Allen RH: Neuropsychiatric disorders caused by cobalamin deficiency in the absence of anemia or macrocytosis. N Engl J Med 1988;318:1720-1728.

-27 Nilsson M, Norberg B, Hultdin J, Sandstrom H, Westman G, Lökk J: Medical intelligence in Sweden. Vitamin B12: oral compared with parenteral? Postgrad Med J 2005;81:191-193.

$\checkmark 28$ Berlin H, Berlin R, Brante G: Oral treatment of pernicious anemia with high doses of vitamin B12 without intrinsic factor. Acta Med Scand 1968;184:247-258.

-29 Eussen SJ, de Groot LC, Clarke R, Schneede J, Ueland PM, Hoefnagels WH, van Staveren WA: Oral cyanocobalamin supplementation in older people with vitamin B12 deficiency: a dose-finding trial. Arch Intern Med 2005; 165:1167-1172.

-30 Elmståhl S, Persson M, Andrén M, Blabolil V: Malnutrition in geriatric patients: a neglected problem? J Adv Nurs 1997;26:851-855.

-31 Soini H, Routasalo P, Lagstrom H: Characteristics of the Mini-Nutritional Assessment in elderly home-care patients. Eur J Clin Nutr 2004;58:64-70.

- 32 Soini H, Routasalo P, Lagstrom H: Nutritional status in cognitively intact older people receiving home care services - a pilot study. J Nutr Health Aging 2005;9:249-253. 
33 McCall R, Cotton E: The validation of a nursing nutritional assessment tool for use on acute elderly wards. J Hum Nutr Diet 2001;14:137-148.

-34 Chai J, Chu FC, Chow TW, Shum NC, Hui WW: Influence of dental status on nutritional status of geriatric patients in a convalescent and rehabilitation hospital. Int J Prosthodont 2006;19:244-249.

- 35 Cronin-Stubbs D, Beckett LA, Scherr PA, Field TS, Chown MJ, Pilgrim DM, Bennett DA, Evans DA: Weight loss in people with Alzheimer's disease: a prospective population based analysis. BMJ 1997; 314:178-179.

-36 Watanabe F, Abe K, Fujita T, Goto M, Hiemori M, Nakano Y: Effects of microwave heating on the loss of vitamin B(12) in foods. J Agric Food Chem 1998;46:206-210.

-37 Ingenbleek Y, Hardillier E, Jung L: Subclinical protein malnutrition is a determinant of hyperhomocysteinemia. Nutrition 2002;18:40-46.

-38 Rodriguez JJ, Santolaria F, Martinez-Riera A, Gonzalez-Reimers E, de la Vega Prieto MJ, Valls MR, Gaspar MR: Clinical significance of homocysteine in elderly hospitalized patients. Metabolism 2006; 55:620-627.

-39 Schulz RJ: Homocysteine as a biomarker for cognitive dysfunction in the elderly. Curr Opin Clin Nutr Metab Care 2007;10:718-723.

-40 Engbersen AM, Franken DG, Boers GH, Stevens EM, Trijbels FJ, Blom HJ: Thermolabile 5,10-methylenetetrahydrofolate reductase as a cause of mild hyperhomocysteinemia. Am J Hum Genet 1995; 56:142-150.

-41 Böttiger AK, Hagnelius NO, Nilsson TK: Mutations in exons 2 and 3 of the FOLR1 gene in demented and non-demented elderly subjects. Int J Mol Med 2007;20:653-662.

42 Nilsson TK, Laanpere M, Altmae S, Serra-Majem L, Salumets A: A folate receptor alpha double-mutated haplotype 1816delC-1841A is distributed throughout Eurasia and associated with lower erythrocyte folate levels. Mol Biol Rep 2012;39:4471-4478.

-43 Ravaglia G, Forti P, Maioli F, Martelli M, Servadei L, Brunetti N, Porcellini E, Licastro F: Homocysteine and folate as risk factors for dementia and Alzheimer disease. Am J Clin Nutr 2005;82:636-643.

-44 Haan MN, Miller JW, Aiello AE, Whitmer RA, Jagust WJ, Mungas DM, Allen LH, Green R: Homocysteine, B vitamins, and the incidence of dementia and cognitive impairment: results from the Sacramento Area Latino Study on Aging. Am J Clin Nutr 2007;85:511-517.

45 Ueland PM, Schneede J: [Measurement of methylmalonic acid, homocysteine and methionine in cobalamin and folate deficiencies and homocysteinuria]. Tidsskr Nor Laegeforen 2008;128:690-693.

-46 Nes M, Sem SW, Rousseau B, Bjorneboe GE, Engedal K, Trygg K, Pedersen JI: Dietary intakes and nutritional status of old people with dementia living at home in Oslo. Eur J Clin Nutr 1988;42:581593. 